Article

\title{
Covalent Decoration of Cortical Membranes with Graphene Oxide as a Substrate for Dental Pulp Stem Cells
}

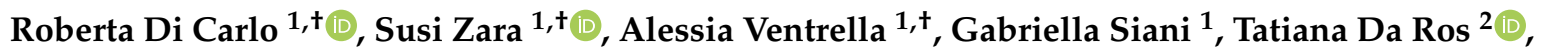 \\ Giovanna Iezzi ${ }^{3}$, Amelia Cataldi ${ }^{1}$ and Antonella Fontana ${ }^{1, *} \mathbb{C}$ \\ 1 Department of Pharmacy, University “G. d'Annunzio", Via dei Vestini, 66100 Chieti, Italy; \\ roberta.dicarlo@unich.it (R.D.C.); susi.zara@unich.it (S.Z.); alessia.ventrella@unich.it (A.V.); \\ gabriella.siani@unich.it (G.S.); amelia.cataldi@unich.it (A.C.) \\ 2 Department of Chemical and Pharmaceutical Sciences, University of Trieste, Piazzale Europa 1, \\ 34127 Trieste, Italy; daros@units.it \\ 3 Department of Medical, Oral and Biotechnological Sciences, University "G. d'Annunzio", Via dei Vestini, \\ 66100 Chieti, Italy; giovanna.iezzi@unich.it \\ * Correspondence: fontana@unich.it; Tel.: +39-0871-3554790 \\ + These authors contributed equally to this work.
}

Received: 29 March 2019; Accepted: 8 April 2019; Published: 12 April 2019

check for updates

\begin{abstract}
Background: The aim of this study was to optimize, through a cheap and facile protocol, the covalent functionalization of graphene oxide (GO)-decorated cortical membrane (Lamina ${ }^{\circledR}$ ) in order to promote the adhesion, the growth and the osteogenic differentiation of DPSCs (Dental Pulp Stem Cells); (2) Methods: GO-coated Laminas were fully characterized by Scannsion Electron Microscopy (SEM) and Atomic Force Microscopy (AFM) analyses. In vitro analyses of viability, membrane integrity and calcium phosphate deposition were performed; (3) Results: The GO-decorated Laminas demonstrated an increase in the roughness of Laminas, a reduction in toxicity and did not affect membrane integrity of DPSCs; and (4) Conclusions: The GO covalent functionalization of Laminas was effective and relatively easy to obtain. The homogeneous GO coating obtained favored the proliferation rate of DPSCs and the deposition of calcium phosphate.
\end{abstract}

Keywords: graphene oxide; covalent functionalization; cortical membranes; calcium phosphate deposition

\section{Introduction}

In this study, we focused our interest on cortical membranes, commonly used in oral surgical procedures, in order to improve their features thanks to a covalent enrichment with graphene oxide (GO). In particular, we used a type of cortical membrane, namely Osteobiol ${ }^{\circledR}$ Lamina provided by Tecnoss. Laminas, created by a registered trademark of Tecnoss, are made up of cortical bone of heterologous origin and are demonstrated to increase the rate of physiological resorption of the material [1]. Laminas have the compactness of bone tissue as well as a flexibility and adaptability that derives from the superficial decalcification process tuned for their preparation [1]. These materials are therefore used to improve bone tissue regeneration in cases in which it is important to reserve a space [2]. It is important to note that these tissue-derived materials are generally brittle and characterized by a low resistance to fracture. These drawbacks were overcome by enriching the original material, i.e., hydroxyapatite, with different additives such as alginate/chitosan [3], titania [4] or carbon nanotubes (CNT) [5]. 
Nowadays, graphene has emerged as a great alternative material for applications in biomedical and regenerative engineering. Graphene is a two-dimensional (2D) carbon-based material which has $s p^{2}$ bonded carbon atoms arranged in a honeycomb lattice structure, with extraordinary electrical, physical, and optical properties [6]. Since its discovery, graphene and its derivatives have been widely investigated for the development of electrical devices and for biomedical applications such as drug delivery systems, biosensors, and regenerative therapies [7]. Mechanically, graphene, despite its flexibility, appears to be one of the strongest materials ever tested. [6] It is transparent, able to conduct electricity and heat better than metals [8], chemically inert, and stable [9]. An increasing number of studies have recently focused on the expansion of new potential applications of graphene nanomaterials, with the aim to highlight the benefits of their use and to improve the application of these nanomaterials [10].

Despite these properties, graphene has a very low solubility in both organic and aqueous solvents. For this reason, hydrophilic graphene derivatives, namely Graphene Oxide (GO), have been widely used and tested for pharmaceutical and biomedical applications. GO is hydrophilic, does not tend to form aggregates, and is highly and homogeneously dispersible in water. GO has been demonstrated to be a biocompatible material whose limited cytotoxicity depends on final concentration, shape, sheet size, dispersibility, and degree of surface functionalization [10]. GO has been investigated for its ability to enhance the proliferation and differentiation of several types of stem cells [11].

The aim of this study was to achieve the covalent functionalization of Laminas, by exploiting, via a simple, cheap, and effective protocol, the capacity of oxygenated groups of GO to interact with cortical membrane surfaces. Indeed, previously investigated GO-coatings [12-14] were obtained by simply depositing GO on the elected substrates and therefore exploiting weak London, Van der Waals, or hydrogen-bonding interactions. The concentrations of GO chosen are those that, in preliminary biological assays and in previous studies $[13,14]$, demonstrated not to be toxic for fibroblast cells and favor osteogenic differentiation in dental pulp stem cells (DPSCs) on collagen membranes. The idea is to demonstrate the ability of graphene oxide to improve Laminas biological properties as well as promote the adhesion, the growth and the osteogenic differentiation of DPSCs (Dental Pulp Stem Cells). DPSCs were chosen because of the easy access to the site collection. Besides, DPSCs have an extensive differentiation ability and their capacity to interact with biomaterials makes them ideal for tissue reconstruction [11].

\section{Materials and Methods}

\subsection{Materials}

Cortical membranes $(0.5 \times 0.5 \times 0.2 \mathrm{~cm})\left(\right.$ Ostebiol ${ }^{\circledR}$ Lamina, Tecnoss) were a gift of Tecnoss dental s.r.l. Pianezza (TO), Italy. GO was purchased from Graphenea, San Sebastian, Spain as an aqueous solution of $4 \mathrm{mg} / \mathrm{mL}$ GO. This solution was diluted at the elected concentration and bath ultrasonicated for $10 \mathrm{~min}$ (Elmasonic $\mathrm{P} 60 \mathrm{H}, 37 \mathrm{kHz}, 180 \mathrm{~W}$ ) before use.

All other reagents were product of analytical grade from Merck KGaA, Darmstadt, Germany and they were used as received.

\subsection{Enrichment with Graphene Oxide}

In order to prepare GO-coated Laminas, a protocol of covalent functionalization was optimized. Firstly, the cortical membranes was activated by using a UV/ozone lamp (PSD-UV4 Novascan UV Ozone System Base model, Novascan Technologies, Boone, NC, USA) for $15 \mathrm{~min}$ on each side. This permits the subsequent coating with the functional groups. Secondly, the Laminas were dipped in $1 \mathrm{M}$ ethanolic solution of 3-aminopropyl triethoxysilane (APTES, commercial sample from Merck KGaA, Darmstadt, Germany) for $3 \mathrm{~h}$ to obtain a thin, stable aminosilane layer on the activated membranes. The so obtained aminosilane-functionalized membranes were rinsed with ethanol and deionized water. Thirdly, these aminosilane-functionalized cortical membranes were dipped in graphene oxide aqueous 
solution of two different concentrations, 5 or $10 \mu \mathrm{g} / \mathrm{mL}$. In particular, $4 \mathrm{~mL}$ of homogenous dispersion of GO in water were added to 10 cortical membranes (ca. $21 \mathrm{mg}$ ) in a baker. The GO solution was left into contact with samples overnight. Finally, membranes were left to dry at room temperature.

Samples were transferred in a 48 multi-well plate for the in vitro tests.

\subsection{Sterilization of Cortical Membranes}

Both pure and GO-coated Laminas were irradiated by using UV irradiation in the Herasafe KS 15, class II, type A2 biological safe cabinet (Thermo Fisher Scientific, North Logan, UT, USA) for $1 \mathrm{~h}$ on each side in order to sterilize the specimens.

\subsection{Apparatus for Chemico-Physical Characterization of Laminas}

Thermo-gravimetric analyses (TGA) were recorded on a TGA Q500 (TA Instruments, New Castle, $\mathrm{DE}, \mathrm{USA}$ ) on ca. $12 \mathrm{mg}$ sample. The runs were performed under nitrogen atmosphere by equilibrating the samples at $100{ }^{\circ} \mathrm{C}$ for $20 \mathrm{~min}$, following a ramp at $10^{\circ} \mathrm{C} / \mathrm{min}$ up to $800^{\circ} \mathrm{C}$.

The morphology of Lamina and GO-coated Laminas was evaluated by Atomic Force Microscopy (AFM), using a Multimode 8 Bruker AFM microscope (Bruker, Milan, Italy) coupled with a Nanoscope $\mathrm{V}$ controller and commercial silicon tips (RTESPA 300, resonance frequency of $300 \mathrm{kHz}$ and nominal elastic constant of $40 \mathrm{~N} \cdot \mathrm{m}^{-1}$ ) with a typical apex radius of $8 \mathrm{~nm}$ in Peak Force and ScanAsyst ${ }^{\mathrm{TM}}$ in air mode.

By using this mode, it was possible, from the height panel, to calculate roughness and, from the force curves recorded at various points, to calculate the Young's modulus. In particular, NanoScope Analysis software 1.8 enables to select the force curves registered at each point of the scanned surface and, from each force curve, to calculate the Young's modulus by fitting the linear part of the retracting curve via a hertzian model. The deflection sensitivity and tip radius were calibrated, prior to use, against standard sapphire.

\subsection{Isolation and Culture of DPSCs}

The Local Ethical Committee of the University "G. d'Annunzio" Chieti-Pescara approved the project (approval number 1173, date of approval 31/03/2016), in agreement with the Declaration of Helsinki. Dental pulps were extracted from third molars derived from young male and female people (age range 18-28 years) which underwent surgical procedures. All patients signed informed consent. The study involved only impacted teeth without dental pathologies. After the extraction, the surrounding tissues were mechanically eliminated and processed as reported in our previous work [15].

Samples were rinsed with phosphate-buffered saline (PBS), maintained in Minimum Essential Medium ( $\alpha$-MEM) (Merck KGaA, Darmstadt, Germany) supplemented with $10 \%$ of Foetal Bovine Serum (FBS) and 1\% antibiotics (penicillin/streptavidin mixture, EuroClone S.p.A, Milan, Italy) and sent to the laboratory for stem cells extraction [15]. When cells covered $80-90 \%$ of the flask area (subconfluence condition) they were subcultured. Antigen expression of CD29, CD45, CD105, CD73 CD90 and SSEA-4 was checked by flow cytometry [15].

\subsection{DPSCs Culture on Laminas}

Cells from the fourth passage (Figure S1 of the Supporting Information) were seeded on Laminas, 10,000 cells $/ \mathrm{cm}^{2}$ were used and cultured up to 28 days. Two hundred twenty Laminas for each experiment were used, fifty-five Laminas were used for each experimental point. Experiments were repeated for three times. At the established times cells were harvested and processed for the required analyses. The cells were cultured in $\alpha$-MEM medium supplemented with differentiation factors such as $10 \mathrm{nM}$ dexamethasone, $0.2 \mathrm{mM}$ ascorbic acid and $10 \mathrm{mM} \beta$-glycerophosphate, as reported elsewhere $[16,17]$. 


\subsection{Scannsion Electron Microscopy (SEM) Analysis}

Samples were fixed with $1.25 \%$ glutaraldehyde in $0.1 \mathrm{M}$ cacodylate buffer for $30 \mathrm{~min}$, dehydrated through alcohol ascending series and then dried with hexamethyldisilazane followed by gold-coating. All micrographs were obtained at $15 \mathrm{kV}$ on compact desktop Phenom XL SEM microscope.

\subsection{Alamar Blue Cell Viability Assay}

The Alamar blue test was performed in triplicate for each experimental sample at each experimental time. Cells viability was measured after 3, 7, 14 and 28 days of culture. The test is based on the reduction of Alamar blue reagent (Thermo Scientific, Rockford, IL, USA), performed only by viable cells, into a red product. At established experimental times the medium was replaced by a new one added with $10 \%$ of Alamar blue reagent, incubated for $4 \mathrm{~h}$ at $37^{\circ} \mathrm{C}$. A spectrophotometric reading at 570 and $600 \mathrm{~nm}$ wavelength was performed. The negative control was established as the value obtained without cells. The percentage reduction of Alamar blue reagent was calculated following the manufacturer instructions.

\subsection{Lactate Dehydrogenase (LDH) Cytotoxicity Assay}

To evaluate biomaterial cytotoxicity, $\mathrm{LDH}$ release into the medium was measured by means of CytoTox 96 non-radioactive cytotoxicity assay (Promega, Madison, WI, USA) at each time point $(3,7$, 14 and 28 days). The LDH leakage in each well was normalized to the lysis value obtained in a lysis well of the same experimental point in which a lysis solution was added to the medium

\subsection{Alizarin Red S (ARS) Staining}

Alizarin red S is a calcium-sensing dye. DPSCs, differentiated towards the osteogenic phenotype, are able to deposit and to induce the mineralization of extracellular matrix rich in calcium phosphate, which can be identified by Alizarin red S. Calcium deposits are detectable for their bright orange-red color.

The DPSCs in each well were rinsed twice with PBS, PBS was discarded and DPSCs were fixed in paraformaldehyde $4 \%$ for $15 \mathrm{~min}$ at room temperature and then washed with deionized water. Alizarin red S staining solution $40 \mathrm{mM}$ (Merck KGaA, Darmstadt, Germany) was added to each well and probed for $20 \mathrm{~min}$ at room temperature (RT) on a shaker. The wells were washed for five times in deionized water. Calcium deposits, stained in orange-red, were dissolved as follows: $10 \%$ acetic acid was added under shaking for $30 \mathrm{~min}$. Laminas were scraped, the liquid containing deposits was collected and vortexed in a tube. Previously heated warm mineral oil (Merck KGaA, Darmstadt, Germany) was added, the tube maintained on ice for $5 \mathrm{~min}$ and eventually centrifuged at 20,000 $g$ for 15 min. The supernatant was discarded and 10\% ammonium hydroxide (Merck KGaA, Darmstadt, Germany) was added. The final solution was analyzed by a spectrophotometric reading performed at $405 \mathrm{~nm}$ wavelength.

\subsection{Statistical Analysis}

SPSS software version 16.0 (SPSS, Inc., Chicago, IL, USA) (Statistical Package for Social Science) and GraphPad Prism 5 were used to perform statistical analysis. Data were evaluated using one-way analysis of variance followed by the Tukey-Kramer post-hoc test. The results were expressed as the mean \pm standard deviation (SD). $P<0.05$ was considered to indicate a statistically significant difference.

\section{Results}

Laminas were enriched with GO at two different concentrations, 5 and $10 \mu \mathrm{g} / \mathrm{mL}$. Photos of the obtained enriched cortical membranes are reported in Figure 1. 


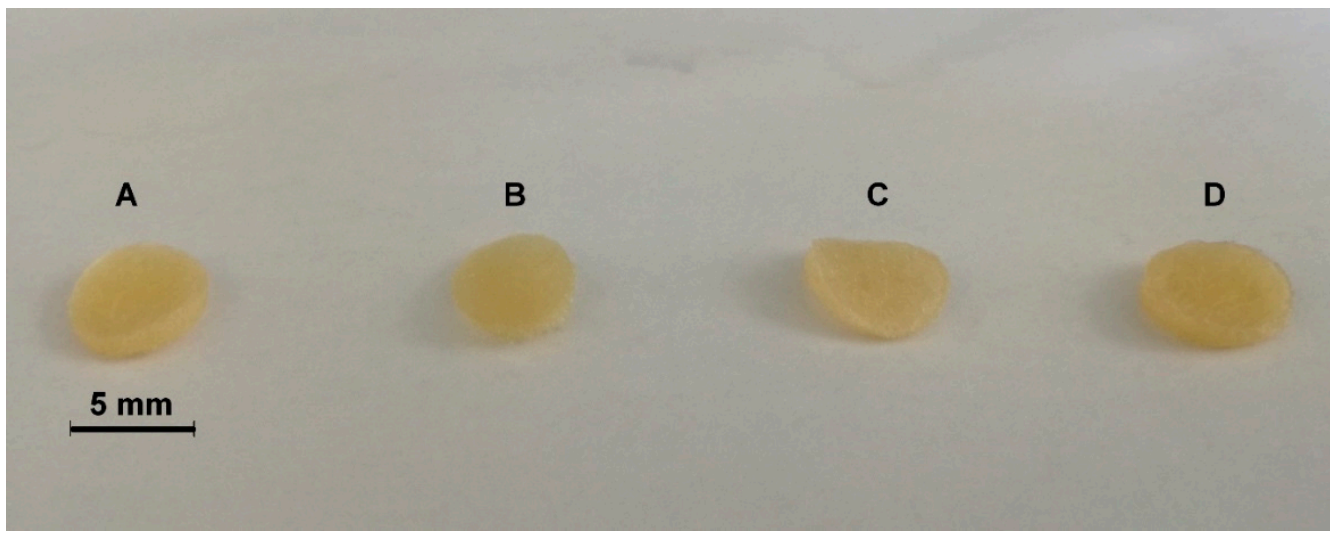

Figure 1. Photographs of (A) pure Lamina, (B) Lamina functionalized with 3-aminopropyl triethoxysilane (APTES) (see Experimental Section 2.2), (C) Lamina enriched with $5 \mu \mathrm{g} / \mathrm{mL}$ graphene oxide (GO) and (D) Lamina enriched with $10 \mu \mathrm{g} / \mathrm{mL} \mathrm{GO.}$

We tried to evaluate the amount of GO covalently attached to the functionalized Lamina by using TGA (Figure 2). While the GO sample presents the common behavior with an important weight loss at $200{ }^{\circ} \mathrm{C}$, the Laminas profiles show a consistent weight loss at around $325^{\circ} \mathrm{C}$ as for $5 \mu \mathrm{g} / \mathrm{mL}$ GO and $10 \mu \mathrm{g} / \mathrm{mL} \mathrm{GO}\left(330^{\circ} \mathrm{C}\right)$, even though for the last preparation a small implement of stability can be appreciated up to $270{ }^{\circ} \mathrm{C}$ with a difference in weight loss of $1.3 \%(9.1 \%$ vs. $10.4 \%$ in the case of control and $5 \mu \mathrm{g} / \mathrm{mL} \mathrm{GO}$ ).

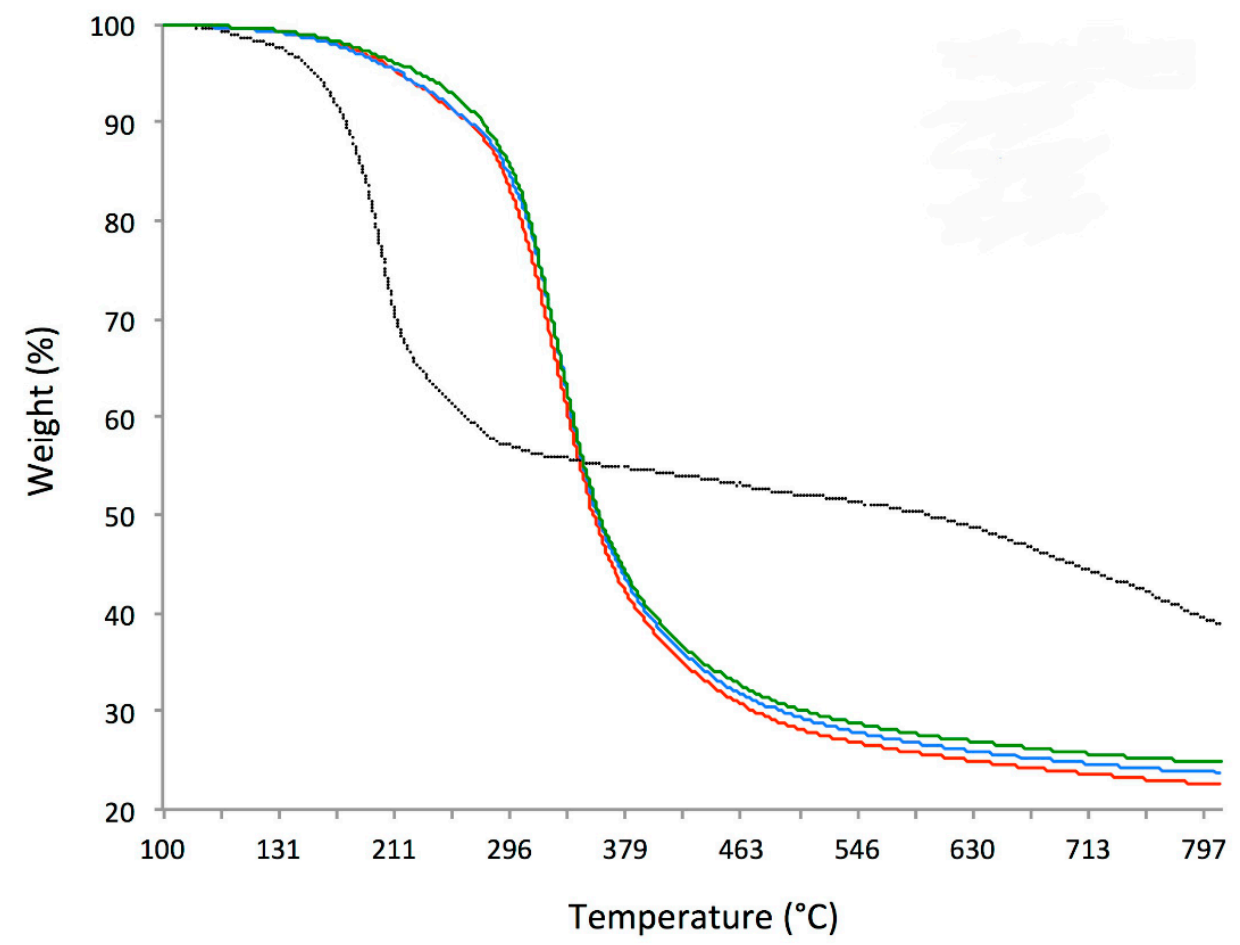

Figure 2. Thermo-gravimetric analyses (TGA) of graphene oxide (black curve), bare Lamina (red curve), $5 \mu \mathrm{g} / \mathrm{mL}$ GO enriched Lamina (blue curve) and Lamina enriched with $10 \mu \mathrm{g} / \mathrm{mL} \mathrm{GO}$ (green curve).

Bare and GO-enriched cortical membranes were analyzed by using AFM. In Figure 3, topographical and tridimensional micrographs as well as peak force error images of bare and GO-enriched $(5 \mu \mathrm{g} / \mathrm{mL}$ and $10 \mu \mathrm{g} / \mathrm{mL}$ ) cortical membranes are reported. From the images reported the changes of the topography of the surface on enrichment with GO are evident. 

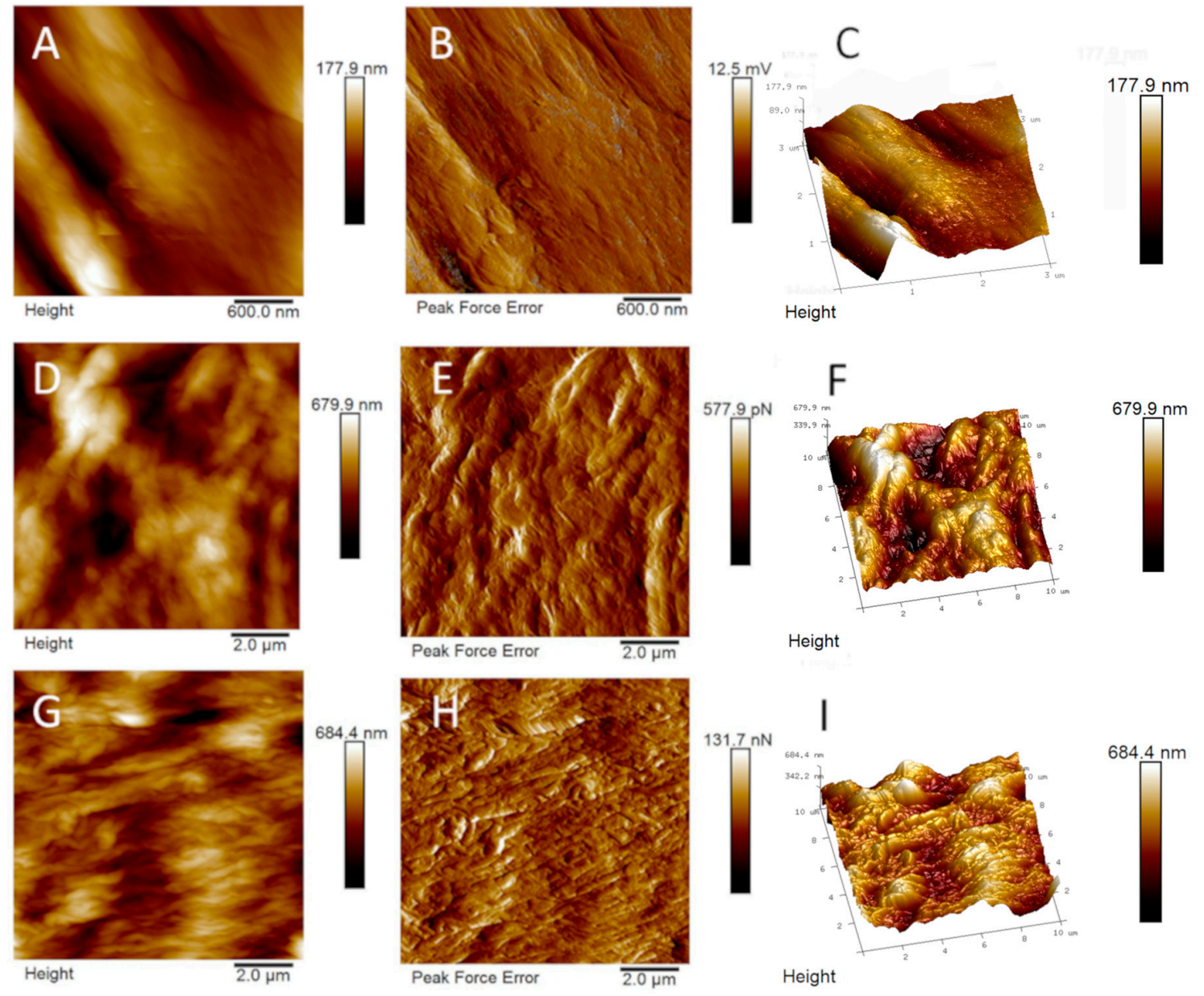

Figure 3. (A,D,G) Topographical, (B,E,H) Peak force error and (C,F,I) Three-dimensional Atomic Force Miscroscopy (AFM) images of bare Lamina (upper line), Lamina enriched with $5 \mu \mathrm{g} / \mathrm{mL}$ of GO (central line) and Lamina enriched with $10 \mu \mathrm{g} / \mathrm{mL}$ GO (bottom line).

From the height panel the Nanoscope analysis 1.8 software (Bruker, Milan, Itay) is able to recover the roughness indexes (i.e., the root-mean square roughness, $\mathrm{Rq}$; the mean absolute value of the surface high deviations, Ra; i.e., the distance between the highest and lowest data points in the image, Rmax; the root-mean square of the surface slope, Sdq, and the ratio between the developed and the planar area, $\mathrm{Sdr}$ ). We calculated these indexes for the bare membrane as the mean values of roughness recovered from two panels with a total surface area of $18 \mu \mathrm{m}^{2}$, were $\mathrm{Rq}=53.0 \pm 10.2 \mathrm{~nm}, \mathrm{Ra}=43.3 \pm 9.3 \mathrm{~nm}$, Rmax $255.5 \pm 20.5 \mathrm{~nm}$, Sdq $12.7 \pm 1.6^{\circ}$, and Sdr $2.5 \pm 0.5 \%$. The roughness indexes, calculated as the mean values recovered from three panels with a total surface area of $300 \mu \mathrm{m}^{2}$, were $\mathrm{Rq}=216.0 \pm 21.2 \mathrm{~nm}$, $\mathrm{Ra}=175.0 \pm 21.9 \mathrm{~nm}, \mathrm{Rmax} 1303.3 \pm 96.3 \mathrm{~nm}, \mathrm{Sdq} 21.4 \pm 1.8^{\circ}$, and Sdr $7.16 \pm 1.0^{\circ}$ for the GO-coated sample with $5 \mu \mathrm{g} / \mathrm{mL}$ and $\mathrm{Rq}=254.7 \pm 56.12 \mathrm{~nm}, \mathrm{Ra}=205.7 \pm 46.5 \mathrm{~nm}, R \max 1311.0 \pm 282.0 \mathrm{~nm}, \mathrm{Sdq}$ $25.9 \pm 10.9^{\circ}$, and Sdr $11.5 \pm 7.6 \%$ for the GO-coated sample with $10 \mu \mathrm{g} / \mathrm{mL}$ (See Supporting Information, Figures S8-S10).

Scansion electron microscopy (SEM) experiments (Figure 4) allowed to observe morphology differences in the investigated Laminas. As seen from SEM images (compare Figures 4B and 4A) the covalent functionalization with amino silane brought about a significant deformation of Lamina Surface. The subsequent coating with GO restored the typical layered structure of GO [13], with layered regions increasing on increasing GO concentration (compare Figures $4 \mathrm{C}$ and $4 \mathrm{D}$ ). 

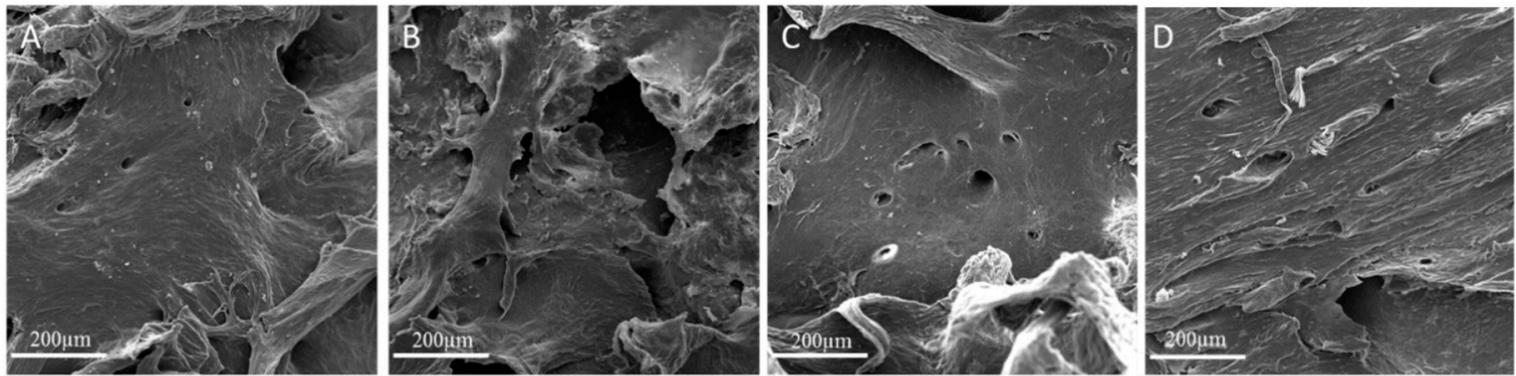

Figure 4. Scansion electron microscopy (SEM) images of (A) bare Lamina, (B) APTES-treated Lamina, (C) $5 \mu \mathrm{g} / \mathrm{mL}$ GO-coated Lamina and (D) $10 \mu \mathrm{g} / \mathrm{mL}$ GO-coated Lamina. Magnification 3000×. Scale bar: $200 \mu \mathrm{m}$.

DPSCs were cultured on Laminas with medium containing differentiation factors up to 28 days; $3,7,14$, and 21 days were chosen as experimental times.

Before starting the evaluation of the biological parameters, an SEM analysis, after 7 and 14 days of culture, was performed in order to evaluate DPSCs morphology, spread and adhesion on Laminas. After 7 days of culture, cells are detectable on all the observed experimental points: DPSCs cultured on control Laminas are flat and spread throughout the surface, some granules of inorganic matrix are starting to be deposited. DPSCs cultured on APTES-treated Laminas appear isolated, with short cytoplasmic extensions, probably suffering and they do not cover all the surface of the Lamina. DPSCs grown on both $5 \mu \mathrm{g} / \mathrm{mL}$ GO- and $10 \mu \mathrm{g} / \mathrm{mL}$ GO-coated Laminas form a uniform cell layer on the biomaterial, they appear completely flat and in close contact with each other; an isolated cell is not recognizable. White granules of inorganic matrix can be identified especially on $5 \mu \mathrm{g} / \mathrm{mL}$ GO-coated Lamina. The same trend is revealed after 14 days of culture (Figure 5).

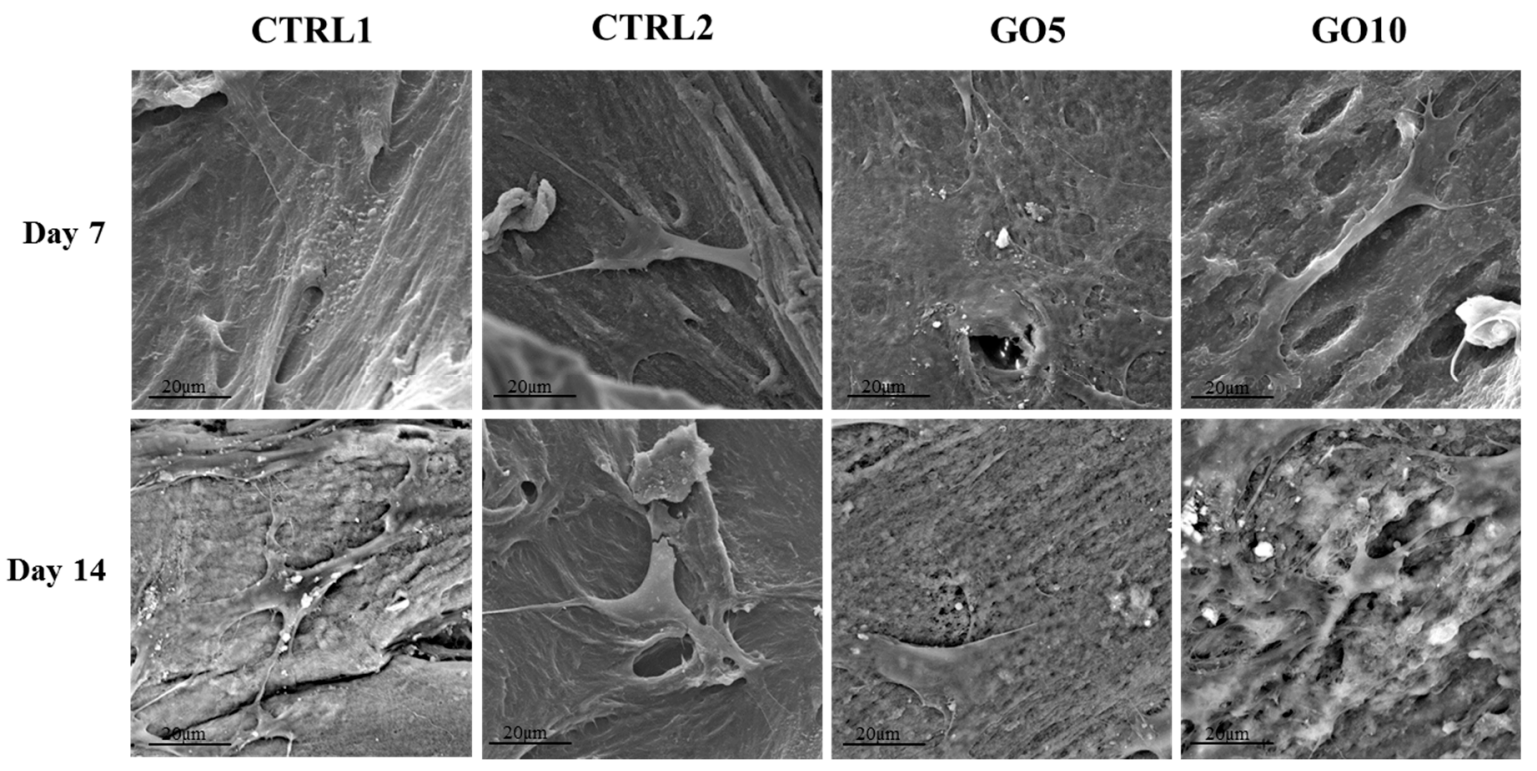

Figure 5. SEM images of Dental Pulp Stem Cells (DPSC)) cultured on bare Laminas (CTRL1), APTES-treated (CTRL2), $5 \mu \mathrm{g} / \mathrm{mL}$ GO-coated (GO5) and $10 \mu \mathrm{g} / \mathrm{mL}$ GO-coated (GO10) Laminas for 7 and 14 days. Magnification $3000 \times$.

Cell viability was measured by means of Alamar Blue Assay after 3, 7, 14, and 28 days. After 3 days of culture the viability level does not show any significant difference among the tested experimental points, whereas after 7 days of culture an appreciable increase in viability level is recordable when DPSCs are cultured on GO-enriched Laminas. In particular, the cell viability is almost doubled for DPSCs cultured on $5 \mu \mathrm{g} / \mathrm{mL}$ GO-coated Laminas with respect to the control and it is comparably high 
for DPSCs cultured on $10 \mu \mathrm{g} / \mathrm{mL}$ GO-coated Laminas. Both the percentage of Alamar Blue reduction recorded on $5 \mu \mathrm{g} / \mathrm{mL}$ GO- and $10 \mu \mathrm{g} / \mathrm{mL}$ GO-coated membranes are statistically significant with respect to the control $(p<0.001)$. The metabolic activity of cell cultured on control and on GO-coated Laminas further augments (Figure 6) until 14 days of culture. By day 14, the number of viable cells reach a plateau, suggesting that those surfaces are advancing into confluence. On the other hand, it is worth noting that the proliferation rate of DPSCs cultured on APTES-treated Laminas is much lower, reaching the maximum percentage of Alamar Blue reduction at 28 days, when the difference with the other samples cancels out.

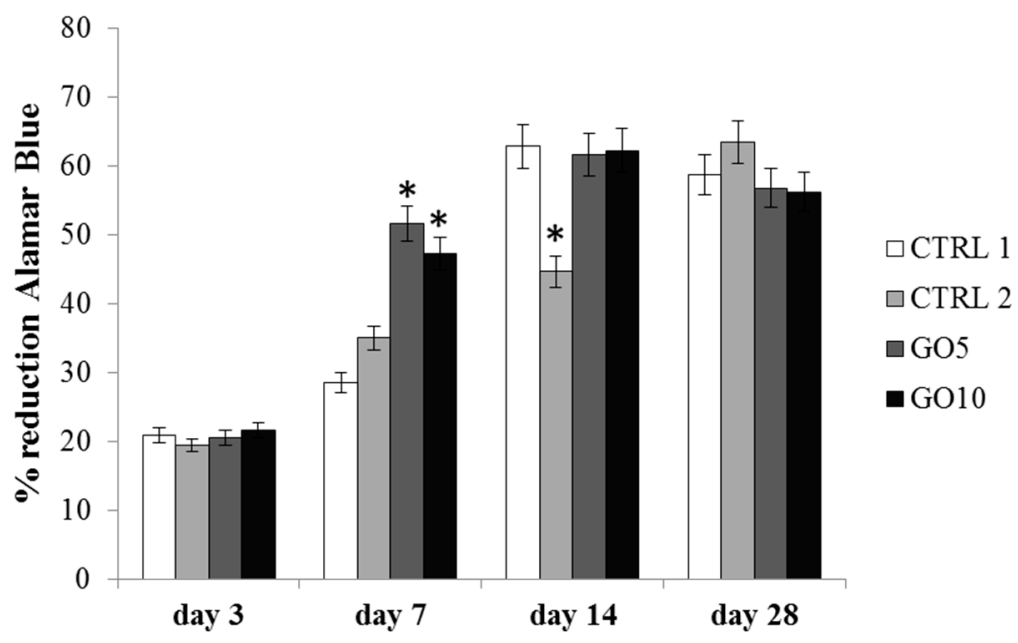

Figure 6. Alamar blue assay in DPSC cultured on bare Laminas (CTRL1), APTES-treated (CTRL2), $5 \mu \mathrm{g} / \mathrm{mL}$ GO-coated (GO5) and $10 \mu \mathrm{g} / \mathrm{mL}$ GO-coated (GO10) Laminas for 3, 7, 14, and 28 days. Forty Laminas were used for each experimental point, ten Laminas per experimental time. The histogram represents Alamar blue reduction percentage, data shown are the mean $( \pm \mathrm{SD})$ of three separate experiments. Zero time \% reduction Alamar Blue: 15.72\%; * Day 7: GO5 and GO10 Laminas vs. control (CTRL1) Laminas $p<0.001$; Day 14 control Laminas, GO5-coated and GO-10 coated Laminas vs. APTES-treated Laminas (CTRL2) $p<0.001$.

The cytotoxicity of the biomaterial was evaluated through LDH assay by measuring the percentage of released LDH within the culture medium after 3, 7, 14, and 28 days of culture. After 3 days of culture the cytotoxicity level is higher than $70 \%$ for all tested samples except for DPSCs cultured on $10 \mu \mathrm{g} / \mathrm{mL}$ GO-coated Laminas. In fact, this sample shows a released LDH percentage significantly lower than that measured for the three others samples. After 7 days of culture the cytotoxicity level starts to decrease with respect to that measured after 3 days of culture in all the investigated samples except for DPSCs cultured on APTES-treated Laminas. In fact, the released LDH percentage for this sample appears still higher than $70 \%$, whereas the percentage decreases under $60 \%$ for cells grown on control Laminas and under $40 \%$ for DPSCs cultured on GO-coated Laminas. The cytotoxicity level does not change thereafter for GO-coated samples and a statistically significant reduction of released LDH percentage is detected for DPSCs grown on $10 \mu \mathrm{g} / \mathrm{mL}$ GO-coated Laminas with respect to the control (Figure 7). Again DPSCs cultured on APTES-treated Laminas show the highest released LDH percentage $(>50 \%)$ among the four Laminas investigated.

Bone matrix deposition was measured through Alizarin Red staining, a calcium-sensing dye able to identify extracellular quantities of calcium phosphate. Alizarin Red staining was performed after 21 and 28 days of culture. After 21 days of culture, a marked decrease of synthetized calcium phosphate could be detected in DPSCs cultured on APTES-treated Laminas compared with the control and with GO-coated Laminas. Conversely, after 28 days of culture, a statistically significant increase in calcium phosphate deposition is detected in DPSCs cultured on $5 \mu \mathrm{g} / \mathrm{mL}$ GO-enriched Laminas with respect to all other tested samples (Figure 8). 


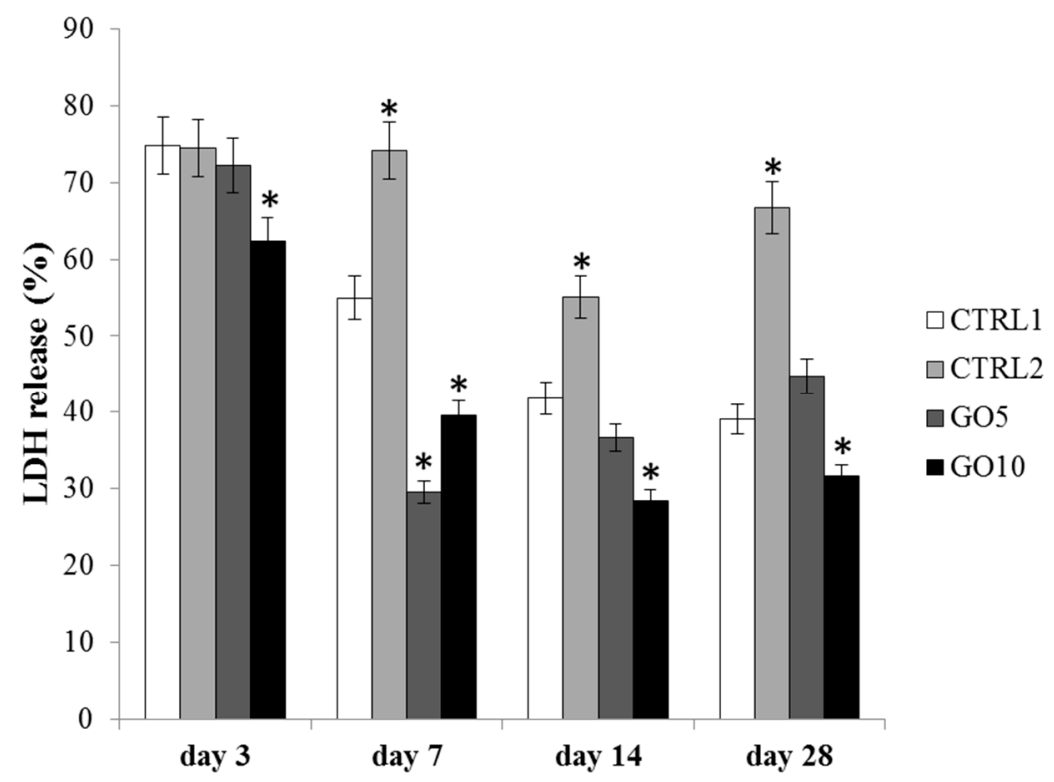

Figure 7. Lactate Dehydrogenase (LDH) assay of DPSC cultured on bare Laminas (CTRL1), APTES-treated (CTRL2), $5 \mu \mathrm{g} / \mathrm{mL}$ GO-coated (GO5) and $10 \mu \mathrm{g} / \mathrm{mL}$ GO-coated (GO10) Laminas for $3,7,14$, and 28 days. Forty Laminas were used for each experimental point, ten Laminas per experimental time. Released LDH is reported as percentage. Data shown are the mean $( \pm \mathrm{SD})$ of three separate experiments. Zero time LDH release (\%): 73.06* Day 3: $10 \mu \mathrm{g} / \mathrm{mL} \mathrm{GO-coated} \mathrm{Laminas}$ (GO10) vs. control (CTRL1) $p<0.05 ;{ }^{*}$ Day 7: APTES-treated, $5 \mu \mathrm{g} / \mathrm{mL}$ GO-coated (GO5) and $10 \mu \mathrm{g} / \mathrm{mL}$ GO-coated Laminas (GO10) vs. control (CTRL1) $p<0.001$; control Laminas, APTES-treated Laminas vs. $5 \mu \mathrm{g} / \mathrm{mL}$ GO-coated Laminas (GO5) $p<0.001$; control Laminas, APTES-treated Laminas vs. $10 \mu \mathrm{g} / \mathrm{mL}$ GO-coated Laminas (GO10) $p<0.001$; * Day 14, day 28: APTES-treated, GO10 Laminas vs. control (CTRL1) Laminas $p<0.005$.

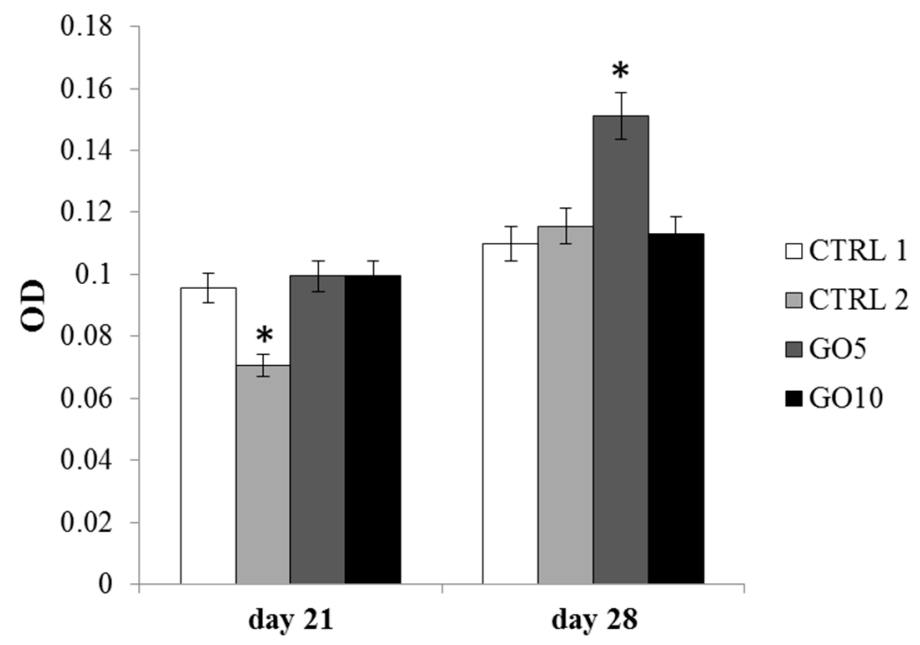

Figure 8. The histogram represents optical density (OD) values of solubilized calcium deposits (orange-red stained) obtained after Alizarin Red staining on bare Laminas (CTRL1), APTES-treated (CTRL2), $5 \mu \mathrm{g} / \mathrm{mL}$ GO-coated (GO5) and $10 \mu \mathrm{g} / \mathrm{mL}$ GO-coated (GO10) Laminas. Twenty Laminas were used for each experimental point, ten Laminas per experimental time. Data shown are the mean $\left( \pm\right.$ SD) of three separate experiments. ${ }^{*}$ Day 21: APTES-treated Laminas vs. control (CTRL1) Laminas $p<0.005$; ${ }^{*}$ Day 28: control Laminas, APTES-treated, $10 \mu \mathrm{g} / \mathrm{mL}$ GO-coated Laminas (GO10) vs. $5 \mu \mathrm{g} / \mathrm{mL}$ GO Laminas (GO5) $p<0.005$. 


\section{Discussion}

Laminas were covalently enriched with GO, by using APTES as the linker between the Lamina and the GO sheets. This type of functionalization was chosen in order to create on the biomaterial a layer of graphene oxide covalently bound to the scaffold. As a matter of fact, in a previous study [12], porcine bone granules, enriched with GO by exploiting simple physical deposition, were implanted in animals for three months and excess GO was detected in the form of GO aggregates in both hard and soft tissues.

TGA did not allow us to properly quantify the amount of GO functionalized onto Laminas, because the amount of GO was very low and the two materials, cortical membrane and GO, evidenced a weight loss at similar temperatures. An approximately 1.3\% GO could be calculated at least for the more concentrated $10 \mu \mathrm{g} / \mathrm{mL}$ sample.

Nevertheless, the GO demonstrated good distribution, through AFM and SEM analyses, on the Lamina and changed completely the appearance of the surface of the cortical membrane. Despite SEM appearance, the enrichment with GO rendered the surface more rough, as already recently evidenced in the case of GO-enrichment of porcine bone granules and collagen membranes [12,13]. Indeed, SEM is not the best technique in order to discriminate GO coverage percentage, but SEM images highlight the formation of layered GO over the Lamina surface (Figure 4). AFM measurements instead evidenced that GO-enriched Laminas are rougher than bare membranes and present a much wrinkled structure (compare panels B, E, and H and C, F, and I in Figure 3).

In particular, the calculated roughness indexes, $\mathrm{Rq}$ and $\mathrm{Ra}$, indicate that the non-coated membrane is characterized by lower peaks and therefore a lower roughness as compared to the GO-coated samples, as confirmed by Rmax values. The surface indexes, Sdr and Sdq, confirmed Ra, Rq, and Rmax data because they evidenced a surface enlargement induced by the presence of GO with more and steep peaks, respectively. Nevertheless no significant differences were highlighted between the samples enriched with 5 or $10 \mu \mathrm{g} / \mathrm{mL} \mathrm{GO}$, likely due to a saturation-like effect of the surface with the lowest concentration of GO investigated.

The measured Young's elastic modulus, obtained as an average value calculated from 20-25 force curves in samples of $10 \mu \mathrm{m} \times 10 \mu \mathrm{m}$ dimensions, is $0.77 \pm 0.46,0.83 \pm 0.62$, and $1.00 \pm 0.27 \mathrm{GPa}$ in bare cortical membrane, $5 \mu \mathrm{g} / \mathrm{mL}$ and $10 \mu \mathrm{g} / \mathrm{mL}$ GO-coated membranes, respectively (See Supporting Information, Figures S2-S7). Therefore it does not change very much on GO enrichment, although a small increase could be monitored on increasing the concentration of GO, indicating that GO contributes to matrix stiffening. It is interesting to note that the SD is very high $(60 \%)$ in the commercial cortical membrane due to the presence of pores and defects, keeps a high value for $5 \mu \mathrm{g} / \mathrm{mL}$ coated Laminas but reduces in Laminas enriched with $10 \mu \mathrm{g} / \mathrm{mL}$ GO, thus highlighting the presence of a homogeneous GO layer in the latter sample. These values are of the same order of those of polyethylene (1.5-2 GPa) and polystyrene (3-3.5 GPa), substrates which have been previously demonstrated to be ideal substrates for the growth of stem cells.

By considering that mesenchymal stem cells demonstrated [18] to sense matrix elasticity and preferentially differentiate depending of the stiffness of the substrate, such values, indicative of stiff matrices, appear proper to favor expression of an osteogenic lineage.

Laminas coated with $5 \mu \mathrm{g} / \mathrm{mL}$ and $10 \mu \mathrm{g} / \mathrm{mL}$ GO were then tested in an in vitro model, by seeding and culturing DPSCs on Laminas surface, in order to evaluate the biocompatibility of GO-enriched Laminas, in terms of cell viability, cytotoxicity, and mineralized matrix deposition.

During the DSPCs differentiation, GO enrichment positively modifies the biological parameters evaluated, thus indicating a good tolerability and an improved biocompatibility. Indeed, GO enrichment, both at 5 and $10 \mu \mathrm{g} / \mathrm{mL}$ concentration, improves the cell spread throughout the surface of the biomaterial thus allowing to hypothesize that $\mathrm{GO}$ enrichment is able to promote the adhesion process and favor the formation of a uniform cell layer (See Figure 5). Moreover, GO coating enhanced the growth rate of DPSCs. In fact, cells seeded on GO-coated Laminas after 7 days of culture show cell viability values two-fold that of bare Laminas evidencing confluence after 14 days of culture (see Figure 6). It is worth 
noting that the above mentioned GO-induced cell viability value is not related to GO concentration as both samples evidence the same effect. Nevertheless it is important to stress that these similarities can be explained by the above mentioned saturation-like effect, with GO covering almost completely the Lamina surface already at the lowest investigated concentration. These results may be associated to the capacity of GO to favor protein adsorption [19]. Indeed, it has been demonstrated that serum proteins absorb quickly and spontaneously to graphene oxide surface to form a corona complex [20] and this adsorption, that demonstrate to be selective for different proteins, may affect adhesion, proliferation, and/or osteogenic differentiation of stem cells [19]. It was also demonstrated that induction of human mesenchymal stem cells (hMSC) differentiation towards different tissue lineages depended on the degree of $\pi-\pi$ stacking with graphene and hydrogen bonding as well as electrostatic interactions with GO [19]. In the present case, a positive effect towards adhesion and viability of DPSCs may be induced also by the highly wrinkled surface associated to a small increase in stiffness obtained on GO enrichment. A similar evidence has been already demonstrated for highly convoluted methacrylate-functionalized GO membrane [21] that favored spontaneous stem cell differentiation towards bone lineage even in the absence of osteogenic growth factors.

The cytotoxicity level also appeared significantly reduced for GO-enriched Laminas during 28 days of culture and actually a statistical significant increase of membrane integrity was detected for Laminas enriched with $10 \mu \mathrm{g} / \mathrm{mL} \mathrm{GO}$ at all the investigated times. It is worth noting that a slightly different behavior characterizes $5 \mu \mathrm{g} / \mathrm{mL}$ GO-coated Laminas, with cytotoxicity slightly increasing and reaching that of the bare Laminas at 14 and 28 days of culture. These results show that, although GO functionalization demonstrated to promote favorable biological effects on DSPCs, it is necessary to carefully tune the concentration of GO bound to Laminas in order to reach the best compromise of effectiveness and biocompatibility. Indeed, different studies evidenced that doses, as well as size, is a fundamental parameter to consider in order to fully characterize GO toxicity [22]. On the other hand, a relatively high toxicity was detected for APTES-treated Laminas. Despite an APTES-treated different material, such as nanoparticles, did not show any toxic effect on cell membrane integrity [23], polyamines demonstrated [24] to promote leakage of liposomal content from 1-palmitoyl-2-oleoyl-sn-glycero-3-phosphocholine (POPC) liposomes due to interactions of primary ammonium groups with phospholipidic head groups. Similarly APTES-treated Laminas could promote analogous ammonium-phospholipidic headgroups interactions, thus explaining the sustained LDH leakage from cell membrane and the chronic cytotoxicity responsible for the lower proliferation rate as compared to cells seeded on the other investigated samples. These data are very interesting because they highlight that, after aminosilane-functionalization, the subsequent treatment with GO allows to override the negative effect evidenced in the presence of APTES-treated Laminas on cell cytotoxicity.

These results are further supported by mineralized bone matrix deposition which appears increased after 28 days of culture and therefore at the end of the osteogenic differentiation [25] but only for DPSCs cultured on $5 \mu \mathrm{g} / \mathrm{mL}$ GO-coated Laminas. They highlight the important role of GO as responsible for a faster and more intense promotion of bone matrix deposition. To conclude, this is a preliminary study on biocompatibility and lack of cytotoxicity of the GO-functionalized Laminas and further investigations are needed in order to fully characterize their biological properties. First of all a detailed investigation focused on the tuning of GO concentration needed for the covalent functionalization of Laminas will allow to optimize the risk-to-benefit balance and clarify all the factors affected by GO enrichment.

\section{Conclusions}

This study demonstrated that the relatively homogeneous coating of investigated commercial cortical membranes with GO was relatively easy to obtain. It favored the proliferation rate of DPSCs probably due to the capacity of GO to adsorb proteins present in the medium. Clear evidences of reduced toxicity were evidenced and Laminas enriched with GO $5 \mu \mathrm{g} / \mathrm{mL}$ demonstrated a statistical significant increase of calcium phosphate deposition. The present study is particularly promising and 
we believe that this material holds potential as useful substrate to facilitate in vivo bone regeneration. Nevertheless it highlights the need to further investigate GO-coated samples in order to tune the concentration of GO that demonstrates the best osteogenic activity and biocompatibility.

Supplementary Materials: The following are available online at http://www.mdpi.com/2079-4991/9/4/604/s1, Figure S1: DPSCs observed with a light microscope before detachment and seeding for osteoblastic differentiation, Figures S2-S7: Original AFM micrographs and representative force curves of pure Lamina, Lamina enriched with $5 \mu \mathrm{g} / \mathrm{mL}$ GO and $10 \mu \mathrm{g} / \mathrm{mL}$ GO used for the mechanical studies, Figures S8-S10: Original AFM micrographs used for the roughness index calculation of pure Lamina, Lamina enriched with $5 \mu \mathrm{g} / \mathrm{mL}$ GO and $10 \mu \mathrm{g} / \mathrm{mL}$ GO, Tables S1-S3: Roughness indexes for pure Lamina, Lamina enriched with $5 \mu \mathrm{g} / \mathrm{mL} \mathrm{GO}$ and $10 \mu \mathrm{g} / \mathrm{mL}$ GO.

Author Contributions: R.D.C. enriched the Laminas with GO, S.Z. performed biological analyses, A.V. performed AFM measurements, G.S. and G.I. data curation and supervision, T.D.R. performed TGA analyses, A.C. and A.F. conceptualization, writing original draft, and supervision.

Funding: This work was carried out with the financial support from the University 'G. d'Annunzio' of Chieti-Pescara and MIUR.

Conflicts of Interest: The authors declare no conflict of interest.

\section{References}

1. Osteobiol by Tecnoss. Available online: https://www.osteobiol.com (accessed on 15 December 2018).

2. Rossi, R.; Rancitelli, D.; Poli, P.P.; Rasia Dal Polo, R.; Nannmark, U.; Maiorana, C. The use of collagenated porcine cortical lamina in the reconstruction of alveolar ridge defects. A clinical and histological study. Minerva Stomatol. 2016, 65, 257-268.

3. Jianguo, L.; Yanqun, L.; Haiyan, L.; Yufen, X.; Yongxiang, Z.; Jingxian, L.; Jianping, W. Preparation, bioactivity and mechanism of nano-hydroxyapatite/sodium alginate/chitosan bone repair material. J. Appl. Biomater. Funct. Mater. 2018, 16, 28-35.

4. Enayati-Jazi, M.; Solati-Hashjin, M.; Nemati, A.; Bakhshi, F. Synthesis and characterization of hydroxyapatite/titania nanocomposites using in situ precipitation technique. Superlattices Microstruct. 2012, 51, 877-885. [CrossRef]

5. Kealley, C.; Elcombe, M.; van Riessen, A.; Ben-Nissan, B. Development of carbon nanotube reinforced hydroxyapatite bioceramics. Physic B 2006, 385-386, 496-498. [CrossRef]

6. Novoselov, K.S.; Fal'ko, V.I.; Colombo, L.; Gellert, P.R.; Schwab, M.G.; Kim, K. A roadmap for graphene. Nature 2012, 490, 192-200. [CrossRef]

7. Kenry, L.W.; Loh, K.P.; Lim, C.T. When stem cells meet graphene: Opportunities and challenges in regenerative medicine. Biomaterials 2018, 155, 236-250. [CrossRef] [PubMed]

8. Balandin, A.A. Thermal properties of graphene and nanostructured carbon materials. Nat. Mater. 2011, 10, 569-581. [CrossRef]

9. Wang, X.L. Proposal for a new class of materials: Spin gapless semiconductors. Phys. Rev. Lett. 2008, 100, 156404. [CrossRef]

10. Guazzo, R.; Gardin, C.; Bellin, G.; Sbricoli, L.; Ferroni, L.; Ludovichetti, F.S.; Piattelli, A.; Antoniac, I.; Bressan, E.; Zavan, B. Graphene-Based Nanomaterials for Tissue Engineering in the Dental Field. Nanomaterials 2018, 8, 349. [CrossRef]

11. Bresson, E.; Ferroni, L.; Gardin, C.; Sbricoli, L.; Gobbato, L.; Ludovichetti, F.S.; Tocco, I.; Carraro, A.; Piattelli, A.; Zavan, B. Graphene based scaffolds effects on stem cells commitment. J. Transl. Med. 2014, 12, 296. [CrossRef] [PubMed]

12. Ettorre, V.; De Marco, P.; Zara, S.; Perrotti, V.; Scarano, A.; Di Crescenzo, A.; Petrini, M.; Hadad, C.; Bosco, D.; Zavan, B.; et al. In vitro and in vivo characterization of graphene oxide coated porcine bone granules. Carbon 2016, 103, 291-298. [CrossRef]

13. De Marco, P.; Zara, S.; De Colli, M.; Radunovic, M.; Lazović, V.; Ettorre, V.; Di Crescenzo, A.; Piattelli, A.; Cataldi, A.; Fontana, A. Graphene oxide improves the biocompatibility of collagen membranes in an in vitro model of human primary gingival fibroblasts. Biomed. Mater. 2017, 12, 055005. [CrossRef]

14. Radunovic, M.; De Colli, M.; De Marco, P.; Di Nisio, C.; Fontana, A.; Piattelli, A.; Cataldi, A.; Zara, S. Graphene oxide enrichment of collagen membranes improves DPSCs differentiation and controls inflammation occurrence. J. Biomed. Mater. Res. Part A 2017, 105, 2312-2320. [CrossRef] 
15. De Colli, M.; Radunovic, M.; Zizzari, V.L.; di Giacomo, V.; Di Nisio, C.; Piattelli, A.; Calvo Guirado, J.L.; Zavan, B.; Cataldi, A.; Zara, S. Osteoblastic differentiating potential of dental pulp stem cells in vitro cultured on a chemically modified microrough titanium surface. Dent. Mater. J. 2018, 37, 197-205. [CrossRef]

16. Guo, T.; Cao, G.; Li, Y.; Zhang, Z.; Nör, J.E.; Clarkson, B.H.; Liu, J. Signals in Stem Cell Differentiation on Fluorapatite-Modified Scaffolds. J. Dent. Res. 2018, 97, 1331-1338. [CrossRef]

17. Xie, H.; Chua, M.; Islam, I.; Bentini, R.; Cao, T.; Viana-Gomes, J.C.; Castro Neto, A.H.; Rosa, V. CVD-grown monolayer graphene induces osteogenic but not odontoblastic differentiation of dental pulp stem cells. Dent. Mater. 2017, 33, e13-e21. [CrossRef]

18. Engler, A.J.; Sen, S.; Sweeney, H.L.; Discher, D.E. Matrix elasticity directs stem cell lineage specification. Cell 2006, 126, 677-689. [CrossRef]

19. Lee, W.C.; Lim, C.H.Y.X.; Shi, H.; Tang, L.A.L.; Wang, Y.; Lim, C.T.; Loh, K.P. Origin of Enhanced Stem Cell Growth and Differentiation on Graphene and Graphene Oxide. ACS Nano 2011, 5, 7334-7341. [CrossRef]

20. Wei, X.-Q.; Hao, L.-Y.; Shao, X.-R.; Zhang, Q.; Jia, X.-Q.; Zhang, Z.-R.; Lin, Y.-F.; Peng, Q. Insight into the Interaction of Graphene Oxide with Serum Proteins and the Impact of the Degree of Reduction and Concentration. ACS Appl. Mater. Interfaces 2015, 7, 13367-13374. [CrossRef]

21. Tang, L.A.; Lee, W.C.; Shi, H.; Wong, E.Y.; Sadovoy, A.; Gorelik, S.; Hobley, J.; Lim, C.T.; Loh, K.P. Highly wrinkled cross-linked graphene oxide membranes for biological and charge-storage applications. Small 2012, 8, 423-443. [CrossRef]

22. Alberto, B. Graphene: Safe or Toxic? The Two Faces of the Medal. Angew. Chem.-Int. Ed. 2013, 52, $4986-4997$.

23. Malvindi, M.A.; De Matteis, V.; Galeone, A.; Brunetti, V.; Anyfantis, G.C.; Athanassiou, A.; Cingolani, R.; Pompa, P.P. Toxicity Assessment of Silica Coated Iron Oxide Nanoparticles and Biocompatibility Improvement by Surface Engineering. PLoS ONE 2014, 9, e85835. [CrossRef]

24. Palermo, E.F.; Lee, D.-K.; Ramamoorthy, A.; Kuroda, K. Role of Cationic Group Structure in Membrane Binding and Disruption by Amphiphilic Copolymers. J. Phys. Chem. B 2011, 115, 366-375. [CrossRef]

25. Riccio, M.; Resca, E.; Maraldi, T.; Pisciotta, A.; Ferrari, A.; Bruzzesi, G.; De Pol, A. Human dental pulp stem cells produce mineralized matrix in 2D and 3D cultures. Eur. J. Histochem. 2010, 54, 205-213. [CrossRef]

(C) 2019 by the authors. Licensee MDPI, Basel, Switzerland. This article is an open access article distributed under the terms and conditions of the Creative Commons Attribution (CC BY) license (http://creativecommons.org/licenses/by/4.0/). 